\title{
Mapa dinâmico com OpenStreetMap e biblioteca Leaflet: Um estudo de caso de mapeamento da COVID-19 no Brasil
}

\author{
Raquel Dezidério Souto \\ Instituto Virtual para o \\ Desenvolvimento Sustentável \\ Atlas Socioeconômico \\ Norte Fluminense \\ Laboratório de Cartografia - \\ GEOCART-UFRJ \\ Rio de Janeiro, Brasil \\ raquel@ivides.org
}

\author{
Danielle Pereira Cintra \\ Atlas Socioeconômico \\ Norte Fluminense \\ Laboratório de Geotecnologias - \\ LAGEOT-UFF \\ Campos dos Goytacazes, Brasil \\ daniellecintra@id.uff.br
}

\author{
Luciana Borges de Oliveira \\ Atlas Socioeconômico \\ Norte Fluminense \\ Laboratório de Geotecnologias - \\ LAGEOT-UFF \\ Campos dos Goytacazes, Brasil \\ oliveiraluciana@id.uff.br
}

\begin{abstract}
We present a case study of the application of the collaborative cartographic base OpenStreetMap and the open JavaScript library Leaflet, in a dynamic map available on the Internet (http://covid19.atlasnf.ivides.org), on the cases of COVID-19 in the North and Northwest Mesoregions of the State of Rio de Janeiro (Brazil). Dynamic mapping is an initiative of the Instituto Virtual para o Desenvolvimento Sustentável (IVIDES.org), in partnership with the Atlas Socioeconômico Norte Fluminense, an extension project of the Universidade Federal Fluminense, sponsored by the Pro-Rectory of Extension (PROEXT-UFF). Data stored in a shapefile (.shp) is extracted and displayed when interacting with the map. The interaction is provided by JavaScript functions from the Leaflet library. Three layers are available - health infrastructure, active positive cases of COVID-19 and confirmed cases of COVID-19. The article includes the main functions used and points out the advantages and disadvantages of applying these technologies in the case in question. There are advantages of using maps of this nature, such as: access to data from anywhere with available Internet and regardless of the type of device or operating system; access to metadata associated with geolocated markers on the map; immediate switching between layers; almost unlimited number of layers. On the other hand, there may be limitations regarding the use of such dynamic maps online by people with little familiarity with new information technologies. With the publication of the article, it's expected to contribute to the use of free software in cartographic applications.
\end{abstract}

Keywords - Dynamic map; OpenStreetMap; Leaflet.

Resumo - Apresentamos um estudo de caso de aplicação da base cartográfica colaborativa OpenStreetMap e a biblioteca JavaScript aberta Leaflet, em um mapa dinâmico disponibilizado na Internet (http://covid19.atlasnf.ivides.org), sobre os casos de COVID-19 nas Mesorregiões Norte e Noroeste Fluminense, no Estado do Rio de Janeiro (Brasil). O mapeamento dinâmico é uma iniciativa do Instituto Virtual para o Desenvolvimento Sustentável (IVIDES.org), em parceria com o Atlas Socioeconômico do Norte Fluminense, um projeto de extensão da Universidade Federal Fluminense, com patrocínio da Pró-Reitoria de Extensão (PROEXT-UFF). Os dados, armazenados em arquivo shapefile (.shp), são extraídos e exibidos no momento da interação com o mapa. A interação é proporcionada por funções JavaScript da biblioteca Leaflet. Três planos de informações são disponibilizados infraestrutura de saúde, casos positivos ativos de COVID-19 e casos confirmados de COVID-19. $O$ artigo inclui as principais funções utilizadas e aponta as vantagens e desvantagens da aplicação dessas tecnologias no caso em questão. As vantagens da utilização de mapas dessa natureza são muitas, tais como: acesso aos dados a partir de qualquer lugar com Internet disponível e independentemente do tipo de dispositivo ou sistema operacional; acesso a metadados associados a marcadores geolocalizados no mapa; alternância imediata entre camadas de informações; número quase ilimitado de camadas. Por outro lado, pode haver limitações no que tange ao uso de tais mapas dinâmicos online por pessoas com pouca familiaridade com novas tecnologias de informação. Além disso, o uso desse tipo de mapa requer a disponibilidade de um equipamento para comunicação via Internet Espera-se, com a publicação do artigo, contribuir para o uso de softwares livres nas aplicações cartográficas.

Palavras-chave — Mapa dinâmico; OpenStreetMap; Leaflet.

\section{INTRODUÇÃO}

O papel das geotecnologias vem ganhando cada vez mais importância, em um mundo globalizado e que compartilha desafios que alcançam a escala planetária. Um exemplo é a pandemia causada pelo retrovírus Sars-Cov-2, cuja doença foi denominada como COVID-19 (sigla da expressão inglesa Coronavirus disease 19). Assim, dispor de sistemas de coleta e exibição de dados online, painéis de sínteses de dados e mapas dinâmicos, torna-se crucial para enfrentar tais crises. No caso de uma pandemia, a geolocalização da informação é fundamental para identificar os pontos de concentração de casos e para acompanhar a disseminação da doença no espaço. 
Os conjuntos de dados geoespacializados - aqueles dados que carregam o atributo da localização espacial - possuem comportamentos próprios à manifestação do fenômeno no espaço. Ou seja, se é um fenômeno caracterizado por uma sequência rápida de eventos, como na disseminação de uma doença durante uma pandemia, o aumento das séries de dados tende a acompanhar a evolução da própria doença. Portanto, fenômenos dessa natureza requerem soluções que acompanhem tal velocidade. Nesse sentido, os Sistemas de Informações Geográficas (SIGs) disponibilizados via Internet, como parte de uma estratégia maior de mapeamento web (web mapping ou webgis), são plataformas a serem consideradas no planejamento e execução de ações relacionadas ao enfrentamento da crise de saúde.

Assim, a informação geoespacial tornou-se alvo de desenvolvimento de um novo campo teórico denominado por "Ciência da geoinformação" (Geoinformation Science ou GIScience). Os estudos acerca da natureza da geoinformação e do alcance dos métodos para sua captura, processamento e exibição (geoprocessamento), passaram a ser então desenvolvidos. Os SIGs passaram a ser abordados sob essa visão e não mais como uma mera "ferramenta" aplicada na pesquisa para um fim maior [1].

Com a evolução dos SIGs, chega-se à novíssima geração destes sistemas, com aplicações disponibilizadas via Internet e que fazem uso de recursos além do hypertext ou hyperlink. Trata-se de uma Internet mais dinâmica e interativa, com valorização dos recursos multimídias e a participação popular, tendo sido apelidada de web 2.0 [2]:

\begin{abstract}
"desenvolvimento da web 2.0 tem causado uma autêntica revolução do ponto de vista comunicativo, ao permitir aos usuários - através das aplicações gratuitas e de fácil manejo, uma participação ativa na rede, oferecendo-lhes a oportunidade de converter-se, ao invés de receptores, em produtores e emissores de seus próprios conteúdos, rompendo com a unidirecionalidade própria dos meios tradicionais e abrindo novos canais de comunicação horizontal, em que é possível um verdadeiro feedback entre o emissor e o receptor." (p. 206)
\end{abstract}

A popularização dos programas livres (free softwares) e de código-fonte aberto (open source) contribuiu para a emergência de soluções online mais robustas e seguras e que contam com comunidades de colaboradores no desenvolvimento, o que favorece o rápido aperfeiçoamento dessas aplicações. Um exemplo é a base cartográfica colaborativa OpenStreetMap (OSM) disponível em http://www.openstreetmap.org/. Nela, podem ser dispostas as geoinformações de forma colaborativa e totalmente online. Além disso, as bases OSM podem ser usadas como bases cartográficas em SIGs standalone, que oferecem plugins especificamente para publicação de mapas online, como é o caso do SIG QGIS (https://qgis.org/) e seu plugin QGIS Cloud (https://qgiscloud.com/).

Além da disponibilização via Internet, as aplicações passaram a incorporar funcionalidades voltadas para a interação com o usuário, a fim de proporcionar uma experiência mais ativa. Nessa via, a biblioteca JavaScript Leaflet (http://www.leaflet.com) oferece diversos recursos para o programador, que tornam mais interessante a experiência do usuário de explorar o mapa.
O presente estudo de caso visa mostrar uma solução, criada com OSM e a biblioteca JavaScript Leaflet, que disponibiliza um mapa dinâmico via Internet, com dados sobre a COVID-19 em municípios das mesorregiões Norte e Noroeste Fluminense, no estado do Rio de Janeiro (Brasil). Com a divulgação dessa pesquisa, espera-se contribuir para o desenvolvimento de novas iniciativas de mapeamento dinâmico com uso de software livre.

\section{MAPA DINÂMICO DA COVID-19}

O Mapa dinâmico COVID-19 - Mesorregiões Norte e Noroeste Fluminense - Rio de Janeiro - Brasil é fruto de uma parceria entre o Instituto Virtual para o Desenvolvimento Sustentável - IVIDES.org (http://www.ivides.org) programação do mapa realizada pela Dra. Raquel Dezidério Souto; e o Laboratório de Geotecnologias (Lageot/UFF) e o Atlas Socioeconômico Norte Fluminense - ASNF (http://atlasnf.sites2.uff.br/), que mapeiam os casos de COVID-19 nos municípios das Mesorregiões Norte e Noroeste Fluminense (MRNNOF). O mapeamento é realizado sob a coordenação geral da Profa. Dra. Danielle Pereira Cintra e alimentação de dados na tabela do mapa dinâmico pela graduanda em Geografia Luciana Borges de Oliveira. Essa iniciativa conta ainda com o apoio do Núcleo de Estudos em Economia, Política e Geografia (NEEPG/UFF), do Núcleo de Estudos Rurais e Urbanos (NERU/UFF), PET Ciranda Rural (UFF) e Laboratório de Cartografia da Universidade Federal do Rio de Janeiro (Geocart/UFRJ). A lista completa da equipe de pesquisa do ASNF está disponível em http://atlasnf.sites2.uff.br/atlassocioeconomico-do-norte-fluminense/ e a lista da equipe do Instituto IVIDES.org está disponível em: http://www.ivides.org/index/index.php/equipe.

O monitoramento da incidência de COVID-19 nas MRNNOF, realizado pela equipe do ASNF, levanta dados diariamente dos boletins epidemiológicos dos vinte e dois municípios das MRNNOF, através de sites da internet e redes sociais oficiais das prefeituras municipais e mensalmente dados do Departamento de Informática do Sistema Único e Saúde (DATASUS/MS), descritos no Quadro 1 como variáveis utilizadas. No Instituto Brasileiro de Geografia Estatística (IBGE), obteve-se o TOTAL POPULACIONAL referente aos 22 municípios da MRNNOF, segundo a estimativa populacional de 2019 e a ÁREA TERRITORIAL atualizada, em $\mathrm{km}^{2}$, além do geocódigo IBGE para o município e o shapefile dos municípios do RJ. A densidade demográfica (em hab. $/ \mathrm{km}^{2}$ ) foi calculada para cada município, a partir da razão entre número de habitantes e sua área territorial total. Vale destacar que as orientações divulgadas pelo Ministério da Saúde servem para alinhar as nomenclaturas utilizadas nos casos de COVID-19 na MRNNOF com os demais municípios do país (https://coronavirus.saude.gov.br/).

Para disponibilização dos dados no mapa dinâmico (http://covid19.atlasnf.ivides.org), foram criados três planos de informações (PI), a saber: i) INFRAESTRUTURA DE SAÚDE ii) CASOS POSITIVOS ATIVOS COVID-19 e iii) CASOS CONFIRMADOS COVID-19 (Figura 1). As variáveis incluídas em cada PI estão listadas no Quadro 1. 
QUADRO I

VARIÁVEIS INCLUÍDAS EM CADA PLANO DE INFORMAÇÕES (PI) DO MAPA DINÂMICO DA COVID-19

\begin{tabular}{|l||}
\hline INFRAESTRUTURA EM SAÚDE \\
\hline MESORREGIÃO, GEOCÓDIGO IBGE, \\
CASOS POSITIVOS ATIVOS, \\
CASOS CONFIRMADOS, ÓBITOS, \\
POPULAÇÃO (2019), ÁREA TERRITORIAL ( $\mathrm{Km}^{2}$ ), \\
DENSIDADE DEMOGRÁFICA (hab./Km²), \\
HOSPITAIS ESPECIALIZADOS COVID19, \\
CENTROS DE TRIAGEM COVID19, \\
MÉDICOS PNEUMOLOGISTAS (SUS), \\
MÉDICOS PNEUMOLOGISTAS (PRIVADO), \\
MÉDICOS IMUNOLOGISTAS (SUS), \\
MÉDICOS IMUNOLOGISTAS (PRIVADO), \\
MÉDICOS INFECTOLOGISTAS (SUS), \\
MÉDICOS INFECTOLOGISTAS (PRIVADO), \\
ENFERMEIROS INTENSIVISTAS (SUS), \\
ENFERMEIROS INTENSIVISTAS (PRIVADO), \\
FISIOTERAPEUTAS RESPIRATÓRIOS (SUS), \\
RESPIRADORES, DATA ATUALIZAÇÃO \\
\hline CASOS POSITIVOS ATIVOS COVID-19 \\
\hline CASOS POSITIVOS ATIVOS, POPULAÇÃO (2019), \\
ÁREA TERRITORIAL (Km²), \\
DENSIDADE DEMOGRÁFICA (hab./Km²), \\
HOSPITAIS ESPECIALIZADOS COVID-19, \\
CENTROS DE TRIAGEM COVID-19, \\
MESORREGIÃO, GEOCÓDIGO IBGE, \\
DATA ATUALIZAÇÃO \\
\hline CASOS CONFIRMADOS COVID-19 \\
\hline CASOS CONFIRMADOS, ÓBITOS, \\
ÁREA TERRITORIAL (Km²), \\
DENSIDADE DEMOGRÁFICA (hab./Km²), \\
HOSPITAIS ESPECIALIZADOS COVID-19, \\
CENTROS DE TRIAGEM COVID-19, \\
MESORREGIÃO, GEOCÓDIGO IBGE, \\
DATA ATUALIZAÇÃO \\
\hline
\end{tabular}

Os dados são armazenados em uma tabela dbase (.dbf), que faz parte de um conjunto de arquivos (shapefile), que contém a representação espacial dos polígonos relativos aos municípios das MRNNOF. A tabela Rio_de_Janeiro_CasosCovid.dbf contém os seguintes campos: ID_OBJETO (ID do polígono), NOME (nome do município), MESOREG (mesorregião de localização do município), GEOMETRIA, GEOCODIGO (geocódigo IBGE do município), AREAKM2 (área territorial total do município em $\mathrm{km}^{2}$ ), POPTOTAL (população do município em 2019), HOSPCOVID (número de hospitais especializados para COVID-19 no município), CENTROSTRI (número de centros de triagem de COVID-19 no município), DENSDEMO (densidade demográfica no município), CONFIRMADO (número de casos confirmados de COVID-19 no município), POSATIVO (número de casos positivos ativos de COVID-19 no município), OBT (número de óbitos por COVID-19 no município), DT (data da última atualização), MES_PNEU (número de pneumologistas na rede do SUS no município), MES_IMUN (número de imunologistas na rede do SUS no município), MES_INFE (número de infectologistas na rede do SUS no município), ENFS_IN (número de enfermeiros do SUS no município), FISS_RES (número de fisioterapeutas respiratórios do SUS no município), MEP_PNEU (número de pneumologistas na rede privada no município), MEP_IMUN (número de imunologistas na rede privada no município), MEP_INFE (número de infectologistas na rede privada no município),
ENFP_IN (número de enfermeiros na rede privada no município), FISP_RES (número de fisioterapeutas respiratórios na rede privada no município), RESPIRAD (total de respiradores disponíveis no município) e OBS (observações eventuais).

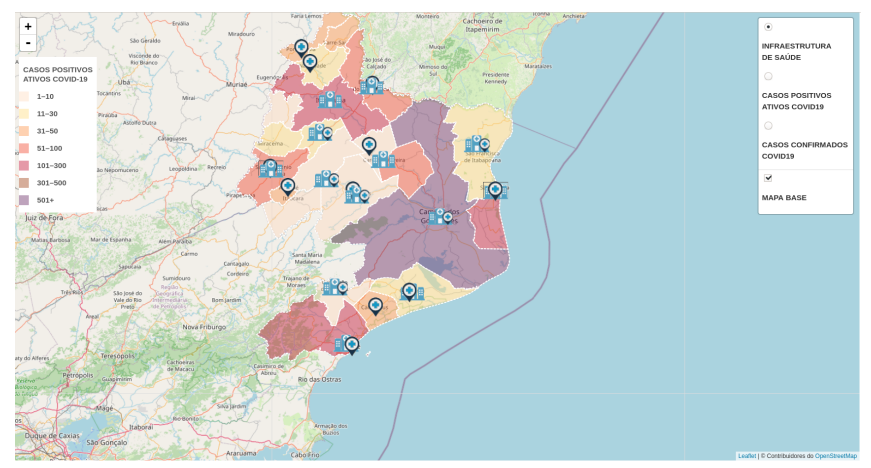

Fig. 1. Planos de informações contemplados no mapa dinâmico.

O shapefile é comprimido em formato .zip, para ser utilizado pela classe Leaflet escrita por Calvin Metcalf, denominada leaflet. shapefile, que faz a extração dos dados do arquivo e os armazena em variáveis que participam da geração e funcionamento do mapa dinâmico. O código da classe leaflet. shapefile pode ser obtido em https:/github.com/calvinmetcalf/leaflet.shapefile. Em seguida, faz-se o upload do arquivo .zip para o diretório de páginas do Instituto IVIDES.org, via protocolo de transferência de arquivos (File Transfer Protocol, FTP).

Para utilizar a biblioteca Leaflet, é necessário indicar os endereços da folha de estilo (Cascade Style Sheet ou CSS) e das redes de distribuição de conteúdo (Content Delivery Networks ou CDN) da Leaflet na seção head do arquivo .html (Figura 2).

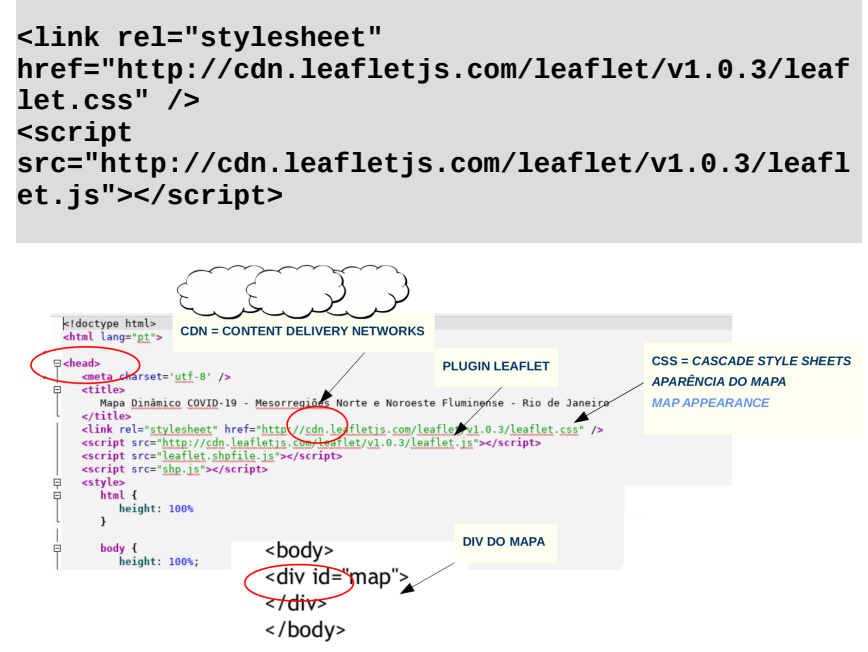

Fig. 2. Elementos básicos para funcionamento do mapa dinâmico.

Além disso, o arquivo do mapa dinâmico (em .html) também foi preparado para carregar a base cartográfica. A base utilizada foi a base colaborativa OpenStreetMap (OSM) - http://www.openstreetmap.org. É necessário incluir uma tag <div id="map"></div> no body do arquivo .html, para criar um contêiner, onde será gerado o mapa (Figura 3). 


\section{/*Criação da variável da camada base*/}

var camada_base =

L.tileLayer ('http://\{s\}.tile.osm.org/ $\{z\} /\{x\} /\{y\} \cdot p$ ng' $^{\prime}$, \{

attribution: '\&copy; Contribuidores do <a href="http://osm.org/copyright">0penStreetMap</a> \})

/ Criação da variável mapa e especificação das coordenadas do centro do mapa e o nível de zoom inicial */

var mapa $=$ L.map ('map', \{layers:

[camada_base] $\}) . \operatorname{setView}([-21.8499,-41.3478])$, v9) ;

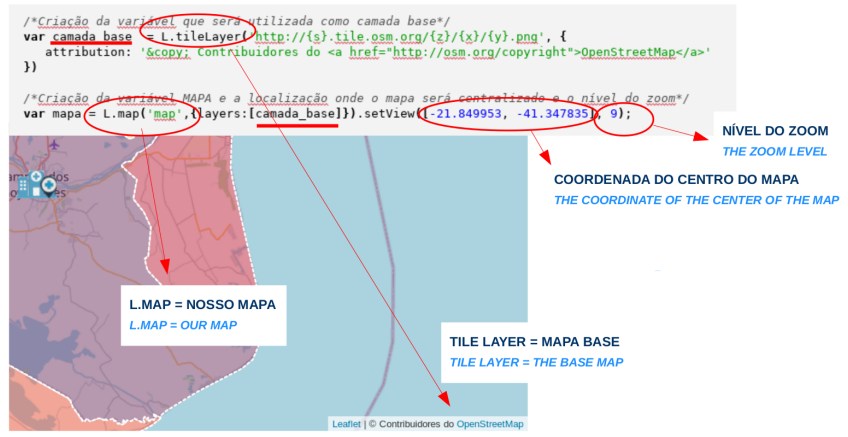

Fig. 3. Elementos fundamentais para a geração do mapa dinâmico.

Uma das bases disponíveis no OpenMapTiles https://openmaptiles.org/, foi utilizada como camada base (base layer). Tais bases cartográficas são validadas por grandes empresas, tais como IBM, Amazon, Siemens ou Bosch, as quais as utilizam. Além disso, devem ser informadas as coordenadas geográficas centrais da primeira exibição do mapa - que, no estudo de caso em questão, foi (21,849953, -41,347835); e o nível de aproximação (Zoom).

Os estilos de mapas OSM são escritos de acordo com a especificação de estilo do MapBox (Mapbox GL Style Specification) e podem ser utilizados em diversos clientes de servidores de mapas, tais como: o próprio Mapbox GL JS (ou OpenLayers), aplicativos móveis para sistemas operacionais iOS ou Android ou em mapas raster (https://openmaptiles.org/styles/).

Dentre as funções da classe leaflet.shapefile, de Calvin Metcalf, foi utilizada a função L. Shapefile para armazenar os dados da tabela .dbf do shapefile em uma variável (Figura 4).

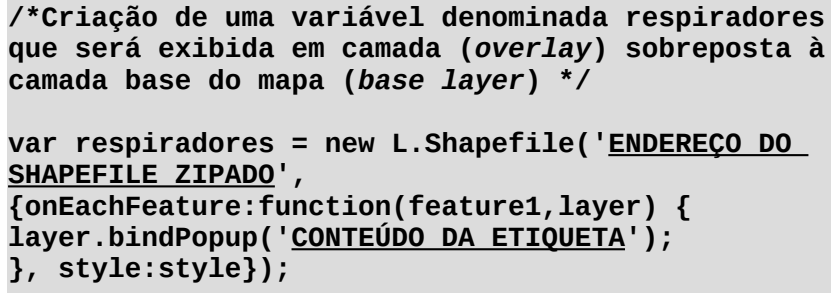

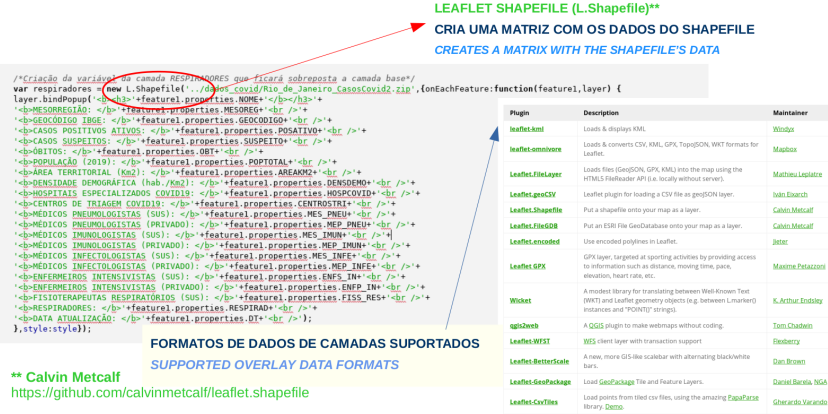

Fig. 4. Função L.Shapefile, utilizada para transferir os dados da tabela para a variável do mapa.

Assim, essa variável armazena os dados em uma matriz, onde cada elemento da matriz corresponde a uma coluna da tabela .dbf. Após a extração dos dados, os valores podem ser utilizados no restante do script do mapa, como, por exemplo, na classificação dos municípios de acordo com o valor da variável e segundo classes de intervalos de valores previamente determinadas. Outros formatos de arquivos de entrada de dados também são suportados pela classe leaflet.shapefile, tais como os formatos Keyhole Markup Language (.kml) ou geoCSV - um arquivo .Csv com extensão espacial, como, por exemplo, o GeoJSON, que inclui atributos espaciais aos registros.

Antes de incluir o controle de camadas (PIs) do mapa dinâmico, é necessário criar, além da camada base (base layer) - que refere-se à base cartográfica utilizada ou mapa base; as camadas referentes aos PIs (overlays), pois essas duas variáveis (base layers e overlays) são atributos da função L.control.layers da classe leaflet . shapefile (Figura 5).

L. control . layers (baseLayers, overlays) . addTo(mapa); camada_base.addTo(mapa); posativos.addTo(mapa);

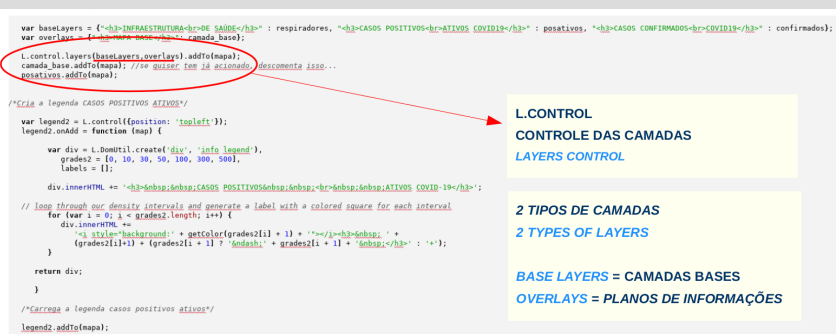

Fig. 5. Controle de camadas (overlays) no mapa dinâmico.

As informações sobre a geolocalização de hospitais especializados e centros de triagem para COVID-19 de cada município são disponibilizadas por meio de ícones dispostos no mapa dinâmico. Os ícones são incluídos com o auxílio da função L.marker, sendo informadas as coordenadas geográficas do ponto como atributos da função (Figura 6).

/*Cria um marcador e plota no mapa, na localização espacial indicada pela coordenadas geográficas */

L.marker ( [-21.13804, -41.67933], \{icon: NOME DA VARIÁVEL\}) . addTo(mapa) . bindPopup ("CONTEÚDO DA ETIQUETA" ); 


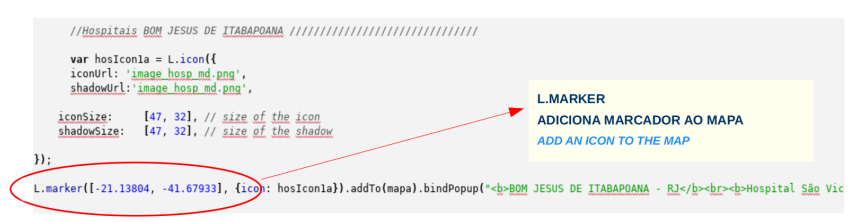

Fig. 6. Inclusão do marcador no mapa dinâmico com a função L.marker.

\section{RESULTADOS E DISCUSSÃO}

Ao visitar o sítio do mapa, http://covid19.atlasnf.ivides.org, o usuário já tem à sua disposição, um mapa temático com a classificação dos municípios das Mersorregiões Norte e Noroeste Fluminense - MRNNOF (Figura 7), de acordo com o valor da variável "casos positivos ativos de COVID-19", nos intervalos de classes: 1-10; 11-30; 31-50; 51-100; 101-300; 301-500 е 500+ (mais de 500 casos). E um controle de PIs, no canto superior direito do mapa. O acesso e utilização do mapa podem ser realizados a partir de qualquer dispositivo com navegador web.

O controle de PIs oferece a opção de escolha dentre três layers: "Infra-estrutura de saúde", "casos positivos ativos de COVID-19" (default) e "casos confirmados de COVID-19". Ao clicar em um município, é disposta uma camada de etiqueta de dados, de acordo com o PI selecionado. $\mathrm{Na}$ Figura 8, mostra-se a etiqueta com as variáveis exibidas no PI "Casos positivos ativos de COVID-19". Já as Figuras 9 e 10 , apresentam as etiquetas para municípios, referentes aos dados dos PIs "Infraestrutura em Saúde" e "Casos confirmados de COVID-19", respectivamente.

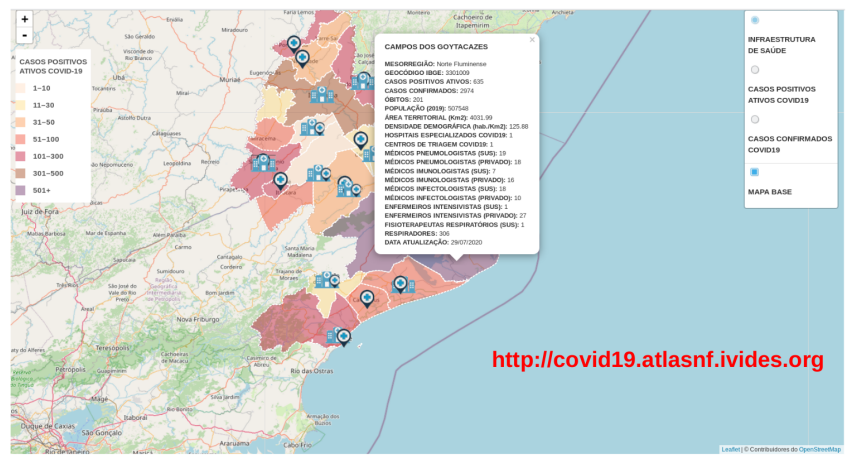

Fig. 7. Controle de camadas e etiqueta de dados no mapa dinâmico.

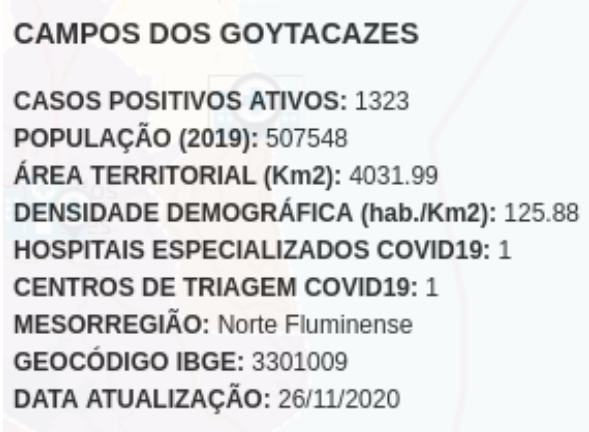

\section{CAMPOS DOS GOYTACAZES}

MESORREGIÃO: Norte Fluminense GEOCÓDIGO IBGE: 3301009 CASOS POSITIVOS ATIVOS: 1323

CASOS CONFIRMADOS: 10650 ÓBITOS: 464

POPULAÇÃO (2019): 507548

ÁREA TERRITORIAL (Km2): 4031.99 DENSIDADE DEMOGRÁFICA (hab./Km2): 125.88 HOSPITAIS ESPECIALIZADOS COVID19: 1 CENTROS DE TRIAGEM COVID19: 1 MÉDICOS PNEUMOLOGISTAS (SUS): 19 MÉDICOS PNEUMOLOGISTAS (PRIVADO): 18 MÉDICOS IMUNOLOGISTAS (SUS): 7 MÉDICOS IMUNOLOGISTAS (PRIVADO): 16 MÉDICOS INFECTOLOGISTAS (SUS): 18 MÉDICOS INFECTOLOGISTAS (PRIVADO): 10 ENFERMEIROS INTENSIVISTAS (SUS): 1 ENFERMEIROS INTENSIVISTAS (PRIVADO): 27 FISIOTERAPEUTAS RESPIRATÓRIOS (SUS): 1 RESPIRADORES: 306

DATA ATUALIZAÇÃO: 26/11/2020

Fig. 9. Etiqueta do PI "Infraestrutura de sáude".

CAMPOS DOS GOYTACAZES
CASOS CONFIRMADOS: 10650
ÓBITOS: 464
POPULAÇÃO (2019): 507548
ÁREA TERRITORIAL (Km2): 4031.99
DENSIDADE DEMOGRÁFICA (hab./Km2): 125.88
HOSPITAIS ESPECIALIZADOS COVID19: 1
CENTROS DE TRIAGEM COVID19: 1
MESORREGIÃO: Norte Fluminense
GEOCÓDIGO IBGE: 3301009
DATA ATUALIZAÇÃO: $26 / 11 / 2020$

Fig. 10. Etiqueta do PI "Casos confirmados COVID-19".

Ao clicar nos marcadores, o visitante acessa o endereço postal completo da unidade de saúde. Foram criados ícones personalizados para distinguir pontos apenas com hospitais, pontos apenas com centros de triagem e pontos onde o hospital inclui um centro de triagem na própria unidade ou anexo (Figura 11).

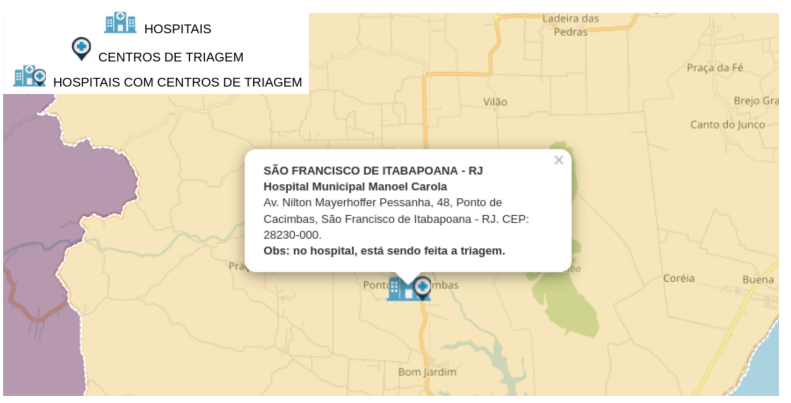

Fig. 11. Ícones de tipos de unidades de atendimento de saúde e etiqueta.

Fig. 8. Etiqueta do PI "Casos positivos ativos COVID-19". 
O link para o mapa dinâmico passou a ser disponibilizado também na seção sobre COVID-19 do portal do Atlas Socioeconômico Norte Fluminense, um projeto de extensão da Universidade Federal Fluminense (UFFCampos), sob a coordenação atual da Profa. Dra. Danielle Pereira Cintra, disponível em http://atlasnf.sites2.uff.br/.

Tanto o mapa dinâmico, quanto os demais produtos do projeto de monitoramento do COVID-19 MRNNOF folheto, painel dinâmico e mapas estáticos, foram disponibilizados amplamente à população residente, agentes de saúde, gestores e demais pesquisadores.

A equipe que colabora com a divulgação dos produtos do ASNF, recebeu considerações de pessoas com pouca familiaridade com novas tecnologias de informação, especialmente pescadores e pescadoras (dada parte da região ser costeira), sobre a dificuldade em usar o mapa dinâmico dos casos de COVID-19. Assim, foram priorizadas formas de divulgação, a depender do público-alvo [3].

\section{CONSIDERAÇÕES FINAIS}

É necessário mencionar que há uma confusão comum entre web mapping (mapa disponível na Internet) e dynamic map (mapa com funcionalidades dinâmicas) e que o presente artigo visou demonstrar a aplicabilidade da conjugação do OpenStreetMap (OSM) e biblioteca JavaScript Leaflet para a geração de mapas dinâmicos e interativos, com o estudo de caso de mapeamento da COVID-19 nas Mesorregiões Norte e Noroeste Fluminense (Rio de Janeiro, Brasil) http://covid19.atlasnf.ivides.org. O mapa está sob licença Creative Commons Attribution-ShareAlike 4.0 International (CC BY-SA 4.0), cujas condições podem ser consultadas em: https://creativecommons.org/licenses/by-sa/4.0/. O códigofonte está disponível em: https://github.com/raqueldeziderio/ mapa.dinamico.

A solução adotada difere de outras soluções para geração de mapas na web. Assim, por exemplo, o painel mantido pelo Ministério da Saúde sobre os casos de COVID-19 no Brasil, disponível em https://susanalitico.saude.gov.br/, foi desenvolvido com a interface de programação de aplicação (Application Program Interface, API) do Google, JSON (formato de arquivo de dados) e AJAX (JavaScript e XML). No entanto, a etiqueta dos dados não é tão detalhada quanto a que é disponibilizada pelo mapa dos casos COVID-19 nas MRNNOF. Além disso, não é possível alternar entre PIs, tendo sido disponibilizados dados de apenas duas variáveis novos casos e novos óbitos. Já o painel de informações sobre COVID-19, mantido pelo Governo do Estado da Bahia, disponível em https://infovis.sei.ba.gov.br/covid19/, adota a mesma solução OSM e Leaflet, porém também disponibiliza etiquetas de dados com poucas variáveis. Além disso, não disponibiliza um controle para troca de PIs

A despeito das diferenças entre as aplicações desenvolvidas, as vantagens da utilização de mapas dinâmicos na web são muitas, tais como: acesso aos dados a partir de qualquer lugar com Internet disponível; acesso aos dados independentemente de sistema operacional ou dispositivo (laptop, tablet, smartphone ou outros); acesso a metadados associados a marcadores geolocalizados no mapa; alternância imediata entre camadas de informações; possibilidade de trabalho com grande número de camadas; código-fonte aberto, o que favorece a personalização das soluções; rapidez no processamento dos dados e desenho do mapa dinâmico, quando da interação com os usuários.

Por outro lado, pode haver limitações no que tange ao uso de tais mapas dinâmicos online por algumas pessoas idosas ou que tem pouca familiaridade com novas tecnologias de informação. Além disso, o uso desse tipo de mapa requer a disponibilidade de um equipamento para comunicação via Internet. Extraordinariamente, a eventual queda do fornecimento de energia elétrica pode também ser um limitador ao acesso, o que não seria problema com a distribuição de folhetos informativos com mapas impressos, por exemplo. Em alguns casos, é necessário oferecer alternativas para comunicação dos resultados, de acordo com algumas características do público-alvo, tais como: faixa etária, poder aquisitivo, nível de escolaridade e disponibilidade de Internet.

A utilização de geotecnologias livres (free softwares) e/ou de código-fonte aberto (open source) também possui suas vantagens, uma vez que possibilita contínuo aperfeiçoamento e diversificação do código fonte (para atender a funcionalidades adicionais). Além disso, em se tratando de aplicações patrocinadas com recursos públicos, a ausência de cobrança de licença de uso é um fator muitas vezes determinante. Com a divulgação dessa iniciativa, espera-se contribuir para o aumento do uso de programas livres em aplicações geoespaciais e elucidar sua aplicabilidade, no caso da programação de um mapa dinâmico disponibilizado na web.

\section{AGRADECIMENTOS}

As autoras agradecem o auxílio à pesquisa que tornaram essa iniciativa possível - Programa de Extensão da PróReitoria de Extensão da Universidade Federal Fluminense (PROEXT-UFF); Programa Nacional de Pós-Doutorado da Coordenação de Aperfeiçoamento de Pessoal de Nível Superior - PNPD-Capes. Como também aos apoiadores do Mapa Dinâmico da COVID-19 Mesorregiões Norte e Noroeste Fluminense: Núcleo de Estudos em Economia Política Geográfica (NEEPG-UFF); Núcleo de Estudos Rurais e Urbanos (NERU-UFF); Pet Ciranda Rural (UFFCampos); e Laboratório de Cartografia (GeoCart-UFRJ).

\section{REFERÊNCIAS}

[1] Wright, D.J. ; Goodchild, M.F. ; Proctor, J.D. Demystifying the Persistent Ambiguity of GIS as 'Tool' versus 'Science'. Annals of the Association of American Geographers. June, 1997.

[2] Subires-Mancera, M.P. Cartografía participativa y web 2.0: Studio de interrelaciones y análises de experiencias. Revista de Comunicación Vivat Academia, n. 117E, diciembre, 2011. pp. 201-2016.

[3] Valente, H.C.M.; Oliveira, L.B.de; Azevedo, L. de A.; Maciel, C.J.N.; Silva, M.T.M.V.; Santos, E.V.M.; Cintra, D.P.; Souto, R.D. Atlas COVID-19 Norte e Noroeste Fluminense: a busca pela melhor compreensão do usuário. V Jornada de Geotecnologias do Estado do Rio de Janeiro - V JGEOTEC. Rio de Janeiro, 09 a 12 de novembro de 2020. 